\title{
Adenocarcinoma de colon sigmoide metastásico en paciente joven de presentación atípica: reporte de caso
}

\author{
An atypical presentation of metastatic sigmoid colon adenocarcinoma in \\ a young patient: case report
}

Kevin Maldonado-Cañón'묘, Edgar Alberto Carmona-Gómez² $\mathbb{D}$

Médico general, Hospital Universitario Nacional de Colombia, Bogotá, D.C., Colombia.

Médico, especialista en Cirugía general y Cirugía de tórax, Hospital Universitario Nacional de Colombia, Bogotá, D.C., Colombia.

\section{Resumen}

El adenocarcinoma colorrectal es la tercera causa de muerte por cáncer en mujeres y la cuarta en hombres a nivel mundial. Se diagnostica en su mayoría en pacientes mayores a los 50 años, siendo la edad media al momento del diagnóstico los 72 años. A pesar eso, se estima que en los próximos años aumentará la incidencia en personas jóvenes y de mediana edad. Debido a esta proyección y considerando que la ausencia de signos y síntomas específicos no permite un diagnóstico oportuno, se hacen necesarias la sensibilización clínica y un alto índice de sospecha en las presentaciones atípicas. Se presenta el caso de una paciente joven que consulta por un cuadro de un mes de síntomas respiratorios quien, después de tratamiento antibiótico, antiviral e inmunomodulador sistémico, presenta sepsis de origen abdominal por peritonitis de cuatro cuadrantes secundaria a perforación de colon sigmoide debida a adenocarcinoma bien diferenciado, metastásico a pulmón, que la llevó a la muerte.

Palabras clave: neoplasias del colon; neoplasias colorrectales; perforación intestinal; peritonitis; metástasis de la neoplasia; adulto joven.

\begin{abstract}
Colorectal adenocarcinoma is the third leading cause of cancer death in women and the fourth in men worldwide. It is diagnosed mostly in patients older than 50 years, being 72 years the mean age at diagnosis. Despite this, it is estimated that the incidence in young and middle-aged people will increase in the coming years. Due to this projection and considering that the absence of specific signs and symptoms does not allow a timely diagnosis, clinical sensitization and a high index of suspicion are necessary in atypical presentations. We present the case of a young
\end{abstract}

Fecha de recibido: 13/11/2019 - Fecha de aceptación: 08/03/2020 - Fecha de publicación en línea: 10/02/2021

Correspondencia: Kevin Maldonado-Cañón, Calle 44 \# 59-75, Subdirección de Investigación, Hospital Universitario Nacional de Colombia, Bogotá, D.C., Colombia. Teléfono: +57 3008747099

Correo electrónico: kmaldonadoc@unal.edu.co

Citar como: Maldonado-Cañón K, Carmona-Gómez EA. Adenocarcinoma de colon sigmoide metastásico en paciente joven, presentación atípica: reporte de caso. Rev Colomb Cir. 2021;36:358-65. https://doi.org/10.30944/20117582.494

Este es un artículo de acceso abierto bajo una Licencia Creative Commons - BY-NC-ND https://creativecommons.org/licenses/by-ncnd/4.0/deed.es 
female patient who consulted for a one-month history of respiratory symptoms who, after treatment with antibiotic, antiviral and systemic immunomodulatory agents, presented sepsis of abdominal origin due to four-quadrant peritonitis secondary to perforation of the sigmoid colon due to adenocarcinoma well differentiated, metastatic to the lung, which led to her death.

Keywords: colonic neoplasms; colorectal neoplasms; intestinal perforation; peritonitis; neoplasm metastasis; young adult.

\section{Introducción}

El cáncer colorrectal es la tercera neoplasia más diagnosticada en el mundo, además es la tercera causa de muerte por cáncer en mujeres y la cuarta en hombres. En Colombia se estima que se diagnosticaron 9140 casos en el 2018, con una tasa de mortalidad de 9,1 por 100.000 habitantes ${ }^{1}$. La edad media al momento del diagnóstico es de 72,4 años y rara vez se presentan antes de los 40 años ${ }^{2}$. En los últimos 20 años, la incidencia en personas menores de 55 años ha aumentado a razón de $2 \%$ por año, y se estima que, en Estados Unidos, para el 2030, el $10 \%$ de los cánceres de colon y el $20 \%$ de los cánceres de recto serán diagnosticados en pacientes menores de 50 años ${ }^{3}$.

Aproximadamente, el 15 a $25 \%$ de los pacientes presentan metástasis a distancia al momento del diagnóstico ${ }^{2,4}$, siendo éste un determinante fundamental para decidir la mejor estrategia terapéutica y el pronóstico de la enfermedad. Las metástasis hepáticas (70 \% en cáncer de colon y $70 \%$ en cáncer de recto) y las metástasis torácicas o pulmonares (32\% en cáncer de colon y $47 \%$ en cáncer de recto) son las más comunes, seguidas por las metástasis al peritoneo ( $21 \%$ en cáncer de colon y $8 \%$ en cáncer de recto) y a hueso (8 \% en cáncer de colon y $12 \%$ en cáncer de recto) ${ }^{2}$. La sobrevida promedio a 5 años es del $60 \%$, sin embargo, una vez se presentan metástasis, la sobrevida a 5 años disminuye al $14 \%{ }^{3}$.

A continuación, se presenta el caso de una paciente joven, quien consulta por síntomas respiratorios y cuyo diagnóstico final fue muerte como consecuencia de cáncer de colon sigmoide perforado con metástasis a pulmón.

\section{Caso clínico}

Se trata de una mujer de 26 años quien consulta por cuadro clínico de un mes de síntomas respira- torios, consistentes en tos seca, rinorrea verdosa, disnea de pequeños esfuerzos (escala de disnea mMRC, del inglés modified Medical Research Council, de 3 puntos), asociados en la semana previa a sensación febril no cuantificada, hiporexia y malestar general. Además, refería pérdida de peso de $3 \mathrm{~kg}$ en el último mes y cambios en el hábito intestinal dados por ausencia de deposiciones en los últimos 5 días. Había recibió manejo antibiótico ambulatorio de primera línea para posible foco pulmonar, con escasa mejoría.

Ingresa estable hemodinámicamente, sin signos de respuesta inflamatoria sistémica, sin dificultad respiratoria, sin agregados pulmonares $\mathrm{u}$ otras particularidades al examen físico. El hemograma de ingreso sin leucocitosis o anemia, solo trombocitosis (455.000 por microlitro). Se consideró inicialmente paciente sintomática respiratoria, con neumonía adquirida en la comunidad por germen bacteriano atípico versus viral; las pruebas para VIH y baciloscopia para tuberculosis fueron negativas.

Se contaba con radiografía de tórax tomada en otra institución en la que se documentaron extensas opacidades parenquimatosas mixtas y ocupación alveolar, involucrando predominantemente los lóbulos inferiores, correspondiente a edema pulmonar evolutivo de origen no cardiogénico. Se decidió tomar tomografía computarizada de tórax de alta resolución (TCAR) (figura 1), cuyos hallazgos anormales se reportaron como consolidaciones bibasales confluentes y en lóbulo medio por probable neumonía multilobar y consolidaciones en lóbulos superiores; no se identificaron masas pulmonares ni derrame pleural, lo cual era concordante con la impresión diagnóstica inicial de neumonía.

Continuó hospitalizada para completar estudios y al decimoprimer día de hospitalización 


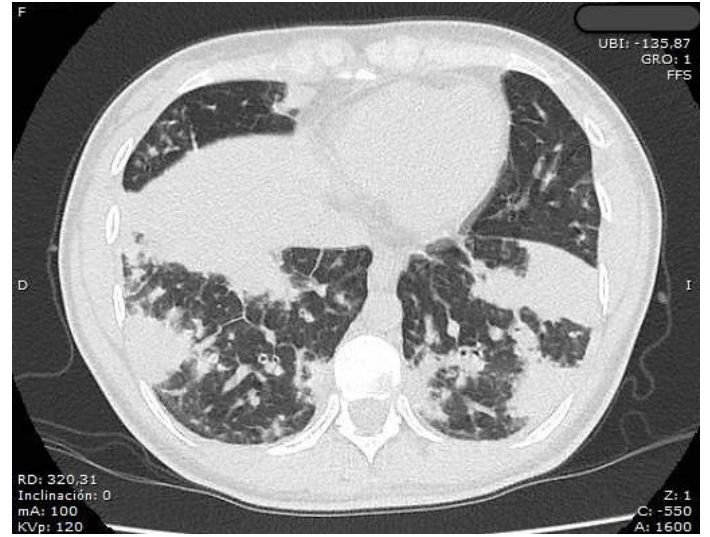

Figura 1. Tomografía de tórax de alta resolución con imagen de consolidaciones bibasales confluentes.

presenta dolor abdominal epigástrico asociado a náuseas, dos episodios eméticos y ausencia de deposiciones, por lo que es valorada por cirugía general quienes solicitan radiografía de abdomen en bipedestación (figura 2), en la que se observa abundante materia fecal en todo el marco cólico por probable estreñimiento, sin signos de obstrucción intestinal, neumoperitoneo, ascitis, ni calcificaciones patológicas, y ecografía de abdomen que reporta leve engrosamiento de las paredes del colon sigmoide, probablemente de origen inflamatorio, e interposición de gas en marco cólico derecho.

Se decidió realizar una tomografía de abdomen (figura 3) que mostró engrosamiento concéntrico de las paredes del colon sigmoide de $7 \mathrm{~mm}$ de espesor, de aspecto inespecífico, de probable etiología inflamatoria, asociado a coprostasis en el colon ascendente y transverso, sin observarse adenomegalias, y endoscopia de vías digestivas altas, cuyo único hallazgo fue gastritis crónica. En la radiografía de tórax de control (figura 4) se aprecian cambios dados por opacidades reticulares bilaterales en 4 cuadrantes y aumento del proceso reticular previo, por lo que se hace necesario la toma de biopsia pulmonar por parte de cirugía de tórax.

Fue llevada a lobectomía segmentaria por toracoscopia (segmento anterior del lóbulo superior derecho y lateral de lóbulo medio) apreciando un pulmón de aspecto enfermo, friable, con múltiples

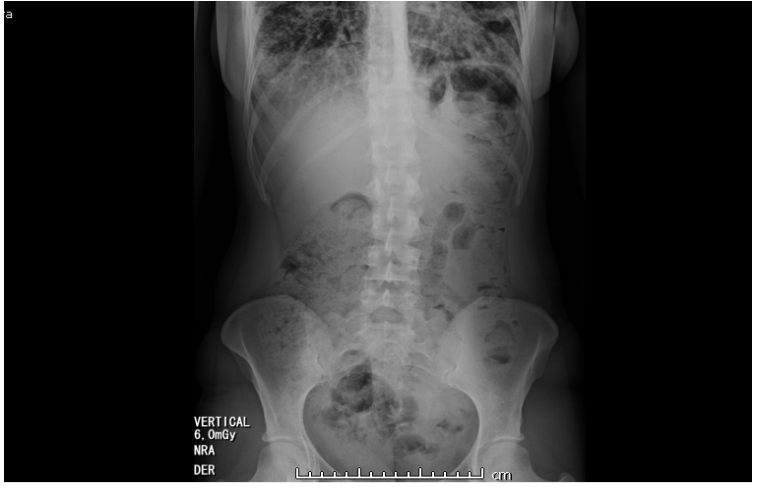

Figura 2. Radiografía simple de abdomen con abundante cantidad de material fecal en el marco cólico por probable estreñimiento, sin signos de obstrucción intestinal ni neumoperitoneo.

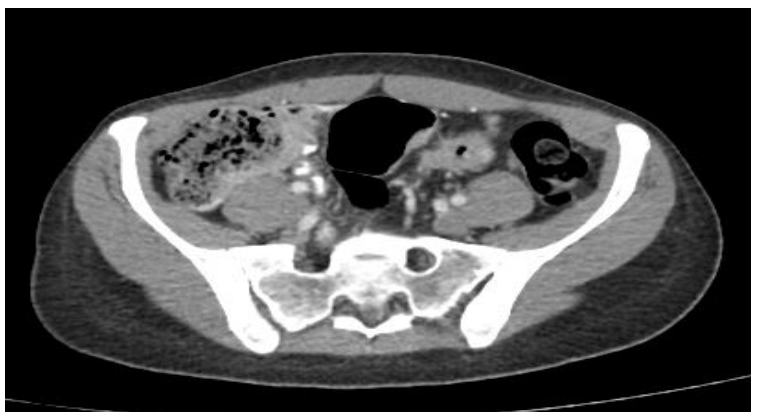

Figura 3. Tomografía de abdomen con imagen de engrosamiento concéntrico de las paredes del colon sigmoide.

lesiones en parches, blanquecinas, sin derrame pleural y sin compromiso de pleura parietal.

Luego de veinte días de hospitalización, presenta dolor abdominal en hemiabdomen inferior, progresivo en intensidad, asociado a tres días de ausencia de deposiciones. Nuevamente es valorada por cirugía general quienes indican manejo sintomático con sonda nasogástrica, líquidos endovenosos y antiespasmódico. La paciente empeora, con taquicardia, disminución en la saturación de oxígeno, defensa abdominal y signos de irritación peritoneal. Bajo sospecha de abdomen agudo, deciden llevar cirugía encontrándose peritonitis fecal de 4 cuadrantes, secundaria a transección completa del colon sigmoides, con salida activa de materia fecal, bordes 

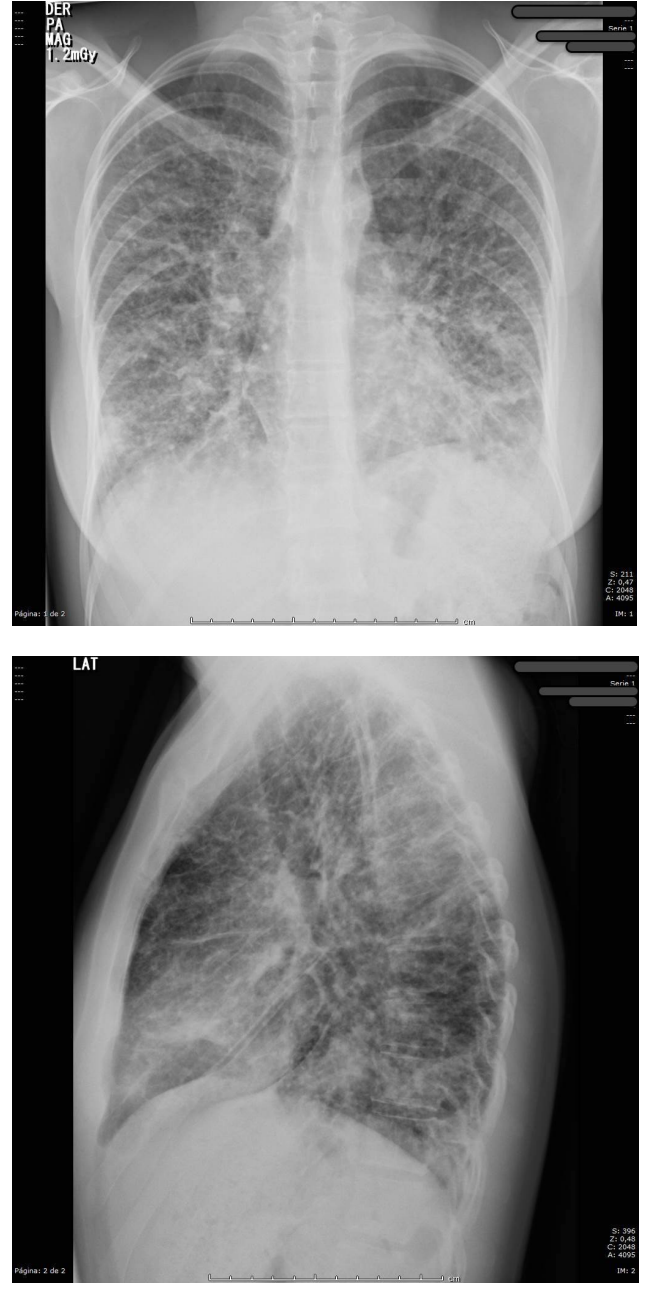

Figura 4. Radiografía de tórax proyección AP y lateral con opacidades reticulares bilaterales en 4 cuadrantes.

severamente inflamados; no se palparon masas en recto, ni se observaron lesiones en hígado o intestino. Se toma muestra del tejido afectado y se realiza sigmoidectomía con colostomía tipo Hartmann vía abierta. Por contaminación fecal severa, se decide el cierre temporal de piel para nuevo lavado quirúrgico en 48 horas.

La paciente es trasladada a la unidad de cuidados intensivos en mal estado general, con soporte ventilatorio, soporte inotrópico a altas dosis y diagnóstico de shock séptico de origen abdominal. A las 48 horas vuelve a cirugía, se hace drenaje de peritonitis residual, lisis de adherencias peritoneales con maniobras romas y lavado peritoneal terapéutico y del tejido celular subcutáneo, dejando bolsa (Viaflex) de interfase entre las asas intestinales y la piel.

Después se recibe el reporte de patología de la biopsia pulmonar con hallazgos de tumor epitelial maligno, interpretado como adenocarcinoma moderadamente diferenciado. Se estudia mediante marcadores de inmunohistoquímica mostrando reactividad para CK20 y CDX2 y negatividad para CK7, TTF1, Napsina A, GATA3, WT1 y PAX 8. En relación con este perfil, la primera alternativa es un adenocarcinoma metastásico, más posiblemente originado en tracto digestivo bajo. Oncología y cuidados intensivos, en conjunto con los familiares, deciden reorientar el esfuerzo terapéutico e iniciar el protocolo de fin de vida. En su trigésimo día de hospitalización presentó asistolia y falleció.

En el reporte de patología de colon sigmoides se describen fragmentos de mucosa colorrectal comprometidos por tumor epitelial maligno ulcerado, con características de adenocarcinomas sincrónicos de tipo intestinal bien diferenciados, con invasión linfovascular, bordes negativos para malignidad y un ganglio linfático del mesenterio positivo para adenocarcinoma metastásico.

\section{Discusión}

Los tumores colorrectales suelen presentar síntomas en los estadios avanzados de la enfermedad. Los tres principales son cambios en el hábito intestinal (74\%), sangrado rectal (51\% en combinación con cambios en el hábito intestinal) y dolor abdominal. Sin embargo, son síntomas comunes, por lo que sólo 5,9\% de los pacientes que presentan uno de estos síntomas tiene, como diagnóstico final, cáncer colorrectal. Entre los pacientes que no presentan estos síntomas principales $(2,6 \%)$, se puede encontrar anemia ferropénica (27\%), masa rectal (7\%) o síntomas perianales $(22 \%)^{5}$.

Las manifestaciones clínicas pueden entenderse a partir de la localización del tumor primario. Tanto los síntomas obstructivos, como el cambio en hábito intestinal, son más comunes en los tumores de colon izquierdo. De la misma forma, la hematoquezia es más común en tumores rectosigmoideos, y los tumores de colon derecho suelen 
manifestarse con síntomas sutiles, como anemia ferropénica y pérdida de peso ${ }^{6}$.

Se entiende como cáncer colorrectal de inicio temprano (en inglés, Early Onset Colorectal Cancer - EOCRC) al tumor que se presenta en pacientes menores a 50 años. La edad media de diagnóstico en este grupo es de 44 años. La incidencia en Estados Unidos por 100.000 habitantes es de 0,85 en pacientes de $20-24$ años y de 28,8 en pacientes de 45-49 años ${ }^{7}$.

La epidemiología en la población colombiana es limitada por el aparente subregistro, sin embargo, se conoce que la mayoría de los pacientes diagnosticados antes de los 50 años de edad, eran mujeres (59\%). Así mismo, se identificaron ciertas diferencias en comparación con los pacientes mayores de 50 años, tales como la historia familiar de cáncer (17\% vs $10 \%$ ), los tumores rectales (49 $\%$ vs $40 \%$ y y la prevalencia de tumores proximales o de colon derecho $(21 \% \text { vs } 30 \%)^{8}$.

En la mayoría de los casos el diagnóstico se hace por los síntomas referidos por el paciente; en menor frecuencia se hace de forma incidental o bajo los programas de tamización, en comparación al grupo etario mayor de 50 años $(5,2 \%$ versus $14,6 \%)^{9}$. Adicionalmente, los pacientes con EOCRC tienen un tiempo más prolongado hasta el diagnóstico en comparación con los pacientes de mayor edad (128 vs 79 días) y síntomas más persistentes ( 60 vs 30 días).

Los tumores en el grupo EOCRC son generalmente más agresivos, acarreando un peor pronóstico pues se diagnostican en un estadio más avanzado (Estadio III y IV) (72 \% vs $63 \%$ ) ${ }^{10,11}$.

En nuestro medio se establecieron los 50 años como la edad a partir de la cual se inicia la tamización para cáncer colorrectal en pacientes sin factores de riesgo, mediante colonoscopia o con la prueba de sangre oculta en materia fecal con la técnica inmunoquímica como alternativa ${ }^{12}$. Ahora bien, en cuanto a los pacientes con sospecha o en riesgo de cáncer colorrectal, se indica igualmente la colonoscopia como método diagnóstico de elección. Es así como se reportó tejido neoplásico en el $10,7 \%$ de las muestras obtenidas en colonoscopia por tamización, el 79,2\% de colonoscopia diagnóstica y el 7,1 \% durante cirugía de urgencia ${ }^{13}$.
En cuanto al estadio al momento de diagnóstico, también se encuentra una relación con el método utilizado, siendo los estadios menos avanzados (estadio I) los más prevalentes en las colonoscopias de tamización (38,5 \%), en comparación con los estadios menos avanzados diagnosticados en colonoscopias diagnósticas $(7,2 \%)$ y cirugía de urgencia $(0 \%)^{13}$.

La presentación de un tumor colorrectal como una emergencia quirúrgica no es rara, a pesar de los avances innegables de tamización y diagnóstico temprano. Se dice que alrededor del $33 \%$ de los pacientes se manifestarán de esta manera, y dentro del espectro clínico se incluyen la obstrucción (hasta en el $77 \%)$, la perforación (2,6 \% a $12 \%$ ) y la hemorragia gastrointestinal aguda con repercusión hemodinámica, que es rara.

Como sucedió con nuestra paciente, la obstrucción es más frecuente en los tumores del colon sigmoide, por su calibre relativamente menor y su contenido de materia fecal sólida ${ }^{14,15}$, y la perforación es la complicación que ocasiona mayor mortalidad (hasta 30-50\%), por estar asociada con peritonitis secundaria y shock séptico consecuente. Dentro de los factores de riesgo para esta complicación, se han descrito la edad avanzada y la hipotensión preoperatoria ${ }^{14,16}$.

Al enfrentarnos a un tumor de colon sigmoide obstructivo, existe una alternativa particularmente útil y es el uso de endoprótesis (o stent), como puente hacia la cirugía electiva, al lograr descomprimir y garantizar una ventana de tiempo prudente, en promedio 7 días ${ }^{17}$. Esto permite corregir una eventual depleción hídrica y desbalances electrolíticos, así como una preparación mecánica del colon fuera del contexto de una emergencia quirúrgica. Aunque la evidencia no es completamente concluyente y su indicación está fundamentada en las condiciones propias del paciente y la experticia del grupo quirúrgico, se reporta que su uso está asociado a una tasa más alta de éxito para la resección y anastomosis primaria (procedimiento de un sólo tiempo) sobre la ostomía (procedimiento de dos tiempos), sin aumentar el riesgo de fuga anastomótica o de absceso intraabdominal, así como a una menor morbilidad general a corto plazo, sin tener 
impacto en la mortalidad, en comparación con la cirugía de emergencia ${ }^{18,19}$.

Respecto al abordaje quirúrgico inicial de un paciente con abdomen agudo no traumático, algunas series de casos apoyan el uso de la laparoscopia sobre la cirugía abierta, argumentando una tasa de éxito del $90 \%$ y un diagnóstico certero en todos los $\operatorname{casos}^{20}$. Así mismo, se reporta un margen apropiado de seguridad y efectividad, equiparable en el abordaje de patologías tales como apendicitis aguda, colecistitis aguda y úlcera péptica perforada, dejando en controversia, por falta de evidencia de alta calidad, su indicación en trauma cerrado o penetrante, obstrucción intestinal y enfermedad diverticular perforada. Se consideran como contraindicaciones relativas los pacientes con inestabilidad hemodinámica, dilatación abdominal marcada, peritonitis fecal y perforaciones secundarias a neoplasias, así es que, la decisión de tomar esta opción depende fundamentalmente de la experiencia particular del grupo quirúrgico con la técnica mínimamente invasiva ${ }^{21}$.

Finalmente, con intención de evaluar las posibilidades que se tienen en los pacientes con heridas quirúrgicas abdominales abiertas, se conoce que en modelos in vitro, al evaluar la presión, las opciones de dispositivos artificiales de cierre difieren entre sí, siendo ABThera y V.A.C. (por sus siglas en inglés, Vacuum-assisted closure) superiores al sistema Barker, y la distribución de presión fue mejor con el sistema V.A.C. frente al resto. Sin embargo, al combinar los parámetros evaluados (entrega y distribución de presión, remoción de fluido y consistencia en el desempeño), se describe el sistema ABThera como el mejor ${ }^{22}$.

Por otro lado, en la práctica in vivo, se recomienda optar por el abdomen abierto en pacientes con peritonitis severa y shock séptico en casos de necesidad para una anastomosis intestinal diferida, un segundo tiempo planeado por isquemia intestinal, falla en el control de la causa de la peritonitis y edema visceral extenso que cause preocupación de síndrome compartimental abdominal. Estos dispositivos permiten un cierre temporal con mecanismos de cierre asistidos por vacío o presión negativa y tracción continua de la fascia como primera opción, dejando como segunda opción las alternativas sin presión negativa (como la bolsa de Bogotá), particularmente en países de bajos recursos, que tienen en un mayor tiempo de cierre y una tasa más alta de fístulas intestinales ${ }^{23}$.

Bajo una revisión retrospectiva del pronóstico y del desenlace de nuestra paciente, lo sucedido concuerda con la información reportada en la literatura. No solo la perforación como manifiesto del tumor colorrectal determina una alta tasa de recurrencia, sino también, se considera que, en pacientes con metástasis a distancia, al momento de la perforación no se puede esperar una sobrevida a largo plazo ${ }^{24}$.

En cuanto a las características histológicas, el adenocarcinoma es la forma más frecuente de cáncer colorrectal, causando más del $90 \%$ de los casos ${ }^{25}$, si bien nuestra paciente difiere a lo reportado en la literatura, al tener en cuenta que son los adenocarcinomas pobremente diferenciados los que más se asocian a perforación. Lo anterior, por supuesto, no excluye la lógica de lo ocurrido en el caso descrito; dentro de los factores asociados como mal pronóstico, al analizar sobrevida y años libre de enfermedad, se encuentran la edad avanzada, pobre diferenciación y tamaño del tumor, estadio avanzado al momento del diagnóstico e invasión venosa y perineural ${ }^{24,26}$.

\section{Conclusión}

Se hace necesario pensar en malignidad cuando nos enfrentemos a un paciente que se presente con una evolución clínica tórpida a pesar del uso de estrategias terapéuticas y ayudas diagnósticas aparentemente bien indicadas. Si bien nuestra paciente y su presentación clínica inicial no la clasificaban, de acuerdo con la evidencia clínica y epidemiológica clásica disponible, como una paciente en alto riesgo de presentar cáncer colorrectal, es importante resaltar el reciente y lento cambio epidemiológico de la neoplasia, por lo que eventualmente se debe redirigir o replantear el análisis clínico, así como la singularidad en la sospecha y los detalles de la evolución natural de este tipo de enfermedad en el paciente joven. 


\section{Cumplimiento de normas éticas}

Consentimiento informado. Se obtuvieron los consentimientos informados y se encuentran disponibles para quienes los requieran. El Comité de Ética Institucional aprobó el diseño y la metodología del estudio.

Conflicto de interés. Los autores certifican que no hay conflicto de intereses

Fuentes de financiación. Este trabajo fue autofinanciado por los autores.

\section{Contribución de los autores:}

- Concepción y diseño del estudio: Kevin MaldonadoCañón.

- Adquisición de datos: Kevin Maldonado-Cañón.

- Análisis e interpretación de datos: Kevin MaldonadoCañón.

- Redacción del manuscrito: Kevin Maldonado-Cañón, Edgar Alberto Carmona-Gómez.

- Revisión crítica: Edgar Alberto Carmona-Gómez.

\section{Referencias}

1. Bray F, Ferlay J, Soerjomataram I, Siegel RL, Torre LA, Jemal A. Global Cancer Statistics 2018: GLOBOCAN estimates of incidence and mortality worldwide for 36 cancers in 185 countries. CA Cancer J Clin. 2018;68:394424. https://doi.org/10.3322/caac.21492.

2. Riihimäki M, Hemminki A, Sundquist J, Hemminki K. Patterns of metastasis in colon and rectal cancer. Sci Rep. 2016;6:29765. https://doi.org/10.1038/srep29765

3. Bailey CE, Hu CY, You YN, Bednarski BK, Rodriguez-Bigas MA, Skibber JM, et al. Increasing disparities in the age-related incidences of colon and rectal cancers in the United States, 1975-2010. JAMA Surgery. 2015;150:1722. https://doi.org/10.1001/jamasurg.2014.1756

4. Van der Geest LGM, Lam-Boer J, Koopman M, Verhoef C, Elferink MAG, de Wilt JHW. Nationwide trends in incidence, treatment and survival of colorectal cancer patients with synchronous metastases. Clin. Exp. Metastasis. 2015;32:457-65.

https://doi.org/10.1007/s10585-015-9719-0

5. Thompson MR, O'Leary DP, Flashman K, Asiimwe A, Ellis BG, Senapati A. Clinical assessment to determine the risk of bowel cancer using Symptoms, Age, Mass and Iron deficiency anaemia (SAMI). BJS Open. 2017;104:1393-1404. https://doi.org/10.1002/bjs.10573

6. Lee GH, Malietzis G, Askari A, Bernardo D, Al-Hassi HO, Clark SK. Is right-sided colon cancer different to left-sided colorectal cancer? - A systematic review. Eur J Surg Oncol. 2015;41:300-8.

https://doi.org/10.1016/j.ejso.2014.11.001
7. Wang R, Wang MJ, Ping J. Clinicopathological features and survival outcomes of colorectal cancer in young versus elderly. A population-based cohort study of SEER 9 registries data (1988-2011). Medicine. 2015;94:e1402. https://doi.org/10.1097/md.0000000000001402

8. Bohorquez M, Sahasrabudhe R, Criollo A, Sanabria-Salas MC, Vélez A, Castro JM, et al. Clinical manifestations of colorectal cancer patients from a large multicenter study in Colombia. Medicine. 2016;95:e4883. https://doi.org/10.1097/MD.0000000000004883

9. Patel SG, Ahnen DJ. Colorectal cancer in the young. Curr Gastroenterol Rep. 2018:20. https://doi.org/10.1007/s11894-018-0618-9

10. Campos FG. Colorectal cancer in young adults: A difficult challenge. World J Gastroenterol. 2017;23:5041-4. https://doi.org/10.3748/wjg.v23.i28.5041

11. Chen FW, Sundaram V, Chew TA, Ladabaum U. Advanced-stage colorectal cancer in persons younger than 50 years not associated with longer duration of symptoms or time to diagnosis. Clin Gastroenterol Hepatol. 2017;15:728-37. https://doi.org/10.1016/j.cgh.2016.10.038

12. Gil-Parada FL, Torres-Amaya M, Riveros-Santoya SV, Castaño-Llano R, Ibáñez H, Huertas-Quintero MM, et al. Guía de práctica clínica para la tamización del cáncer colorrectal - 2015. Rev Colomb Gastroenterol. 2015;30(Suppl 1):67-74.

13. Moreno CC, Mittal PK, Sullivan PS, Rutherford R, Staley $\mathrm{CA}$, Cardona $\mathrm{K}$, et al. Colorectal cancer initial diagnosis: screening colonoscopy, diagnostic colonoscopy, or emergent surgery, and tumor stage and size at initial presentation. Clin Colorectal Cancer. 2016;15:67-73. https://doi.org/10.1016/j.clcc.2015.07.004

14. Baer C, Menon R, Bastawrous S, Bastawrous A. Emergency presentations of colorectal cancer. Surg Clin North Am. 2017;97:529-45. https://doi.org/10.1016/j.suc.2017.01.004

15. Maddu KK, Mittal P, Arepalli CD, Shuaib W, Tewari A, Khosa F. Colorectal emergencies and related complications: A comprehensive imaging review-Noninfectious and noninflammatory emergencies of colon. Am J Roentgenol. 2014;203:1217-29. https://doi.org/10.2214/ajr.13.12323

16. Barnett A, Cedar A, Siddiqui F, Herzig D, Fowlkes E, Thomas CR. Colorectal cancer emergencies. J Gastroint Can. 2013;44:132-42. https://doi.org/10.1007/s12029-012-9468-0

17. Watt AM, Faragher IG, Griffin TT, Rieger NA, Maddern GJ. Self-expanding metallic stents for relieving malignant colorectal obstruction: a systematic review. Ann Surg. 2007;246:24-30.

https://doi.org/10.1097/01.sla.0000261124.72687.72

18. Cirocchi R, Farinella E, Trastulli S, Desiderio J, Listorti C, Boselli C, et al. Safety and efficacy of endoscopic colonic stenting as a bridge to surgery in the management of intestinal obstruction due to left colon and rectal can- 
cer: A systematic review and meta-analysis. Surg Oncol. 2013;22:14-21.

https://doi.org/10.1016/j.suronc.2012.10.003

19. Arezzo A, Passera R, Lo Secco G, Verra M, Bonino MA, Targarona E, Morino M. Stent as bridge to surgery for left-sided malignant colonic obstruction reduces adverse events and stoma rate compared with emergency surgery: results of a systematic review and meta-analysis of randomized controlled trials. Gastrointest Endosc. 2017;86:416-26.

https://doi.org/10.1016/j.gie.2017.03.1542

20. Caruso C, La Torre M, Benini B, Catani M, Crafa F, De Leo $A$, et al. Is laparoscopy safe and effective in nontraumatic acute abdomen? Journal of Laparoendoscopic \& Advanced Surgical Techniques. 2011;21:589-93. https://doi.org/10.1089/lap.2011.0030

21. Navez B, Navez J. Laparoscopy in the acute abdomen. Best Pract Res Clin Gastroenterol. 2014;28:3-17. https://doi.org/10.1016/j.bpg.2013.11.006

22. Delgado A, Sammons A. In vitro pressure manifolding distribution evaluation of ABThera ${ }^{\mathrm{TM}}$ Active Abdominal Therapy System, V.A.C.® Abdominal Dressing System, and Barker's vacuum packing technique conducted under dynamic conditions. SAGE Open Med. 2016;4:205031211562498. https://doi.org/10.1177/2050312115624988

23. Coccolini F, Roberts D, Ansaloni L, Ivatury R, Gamberini E, Kluger Y, et al. The open abdomen in trauma and non-trauma patients: WSES guidelines. World J Emerg Surg. 2018;13. https://doi.org/10.1186/s13017-018-0167-4

24. Asano H, Kojima K, Ogino N, Fukano H, Ohara Y, Shinozuka N. Postoperative recurrence and risk factors of colorectal cancer perforation. Inter J Colorectal Dis. 2017;32:419-24.

https://doi.org/10.1007/s00384-016-2694-3

25. Fleming M, Ravula S, Tatishchev SF, Wang HL. Colorectal carcinoma: Pathologic aspects. J Gastroint Oncol. 2012;3:153-73.

https://doi.org/10.3978/j.issn.2078-6891.2012.030

26. Dai W, Li Y, Meng X, Cai S, Li Q Cai G. Does tumor size have its prognostic role in colorectal cancer? Re-evaluating its value in colorectal adenocarcinoma with different macroscopic growth pattern. Int J Surg. 2017;45:10512. https://doi.org/10.1016/j.ijsu.2017.07.100 